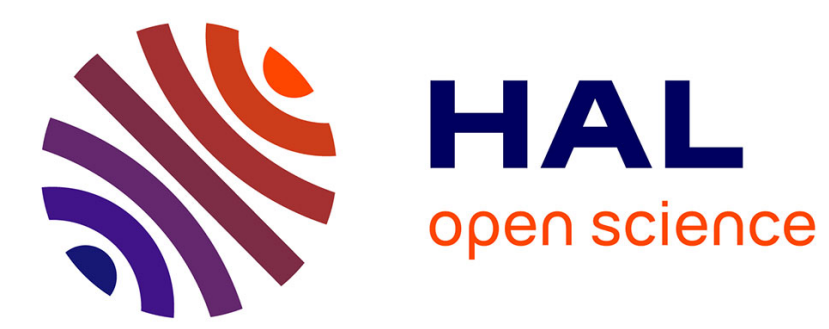

\title{
NON-STATIONARY HYPERSPECTRAL FORWARD MODEL AND HIGH-RESOLUTION
}

\author{
Ralph Abi-Rizk, François Orieux, Alain Abergel
}

\section{To cite this version:}

Ralph Abi-Rizk, François Orieux, Alain Abergel. NON-STATIONARY HYPERSPECTRAL FORWARD MODEL AND HIGH-RESOLUTION. IEEE International Conference on Image Processing 2020 (ICIP 2020), Oct 2020, Abu-Dhabi, United Arab Emirates. hal-02773302

\section{HAL Id: hal-02773302 \\ https://hal.science/hal-02773302}

Submitted on 4 Jun 2020

HAL is a multi-disciplinary open access archive for the deposit and dissemination of scientific research documents, whether they are published or not. The documents may come from teaching and research institutions in France or abroad, or from public or private research centers.
L'archive ouverte pluridisciplinaire HAL, est destinée au dépôt et à la diffusion de documents scientifiques de niveau recherche, publiés ou non, émanant des établissements d'enseignement et de recherche français ou étrangers, des laboratoires publics ou privés. 


\section{NON-STATIONARY HYPERSPECTRAL FORWARD MODEL AND HIGH-RESOLUTION}

\author{
Ralph Abi-rizk*, François Orieux \\ Laboratoire des Signaux et Systèmes (L2S) \\ Université Paris-Saclay - CNRS - CentraleSupelec \\ 3 rue Juliot-Curie, 91912 Gif-sur-Yvette, France
}

\author{
Alain Abergel \\ Institut d'Astrophysique Spatiale (IAS) \\ Université Paris-Saclay - CNRS \\ 91405 Orsay, France
}

\begin{abstract}
We present in this work a forward model for an Integral Field Unit (IFU) instrument. Our model is general but primarily developed for the Mid Resolution Spectrometer of the Mid Infrared Instrument on board the James Webb Space Telescope (JWST). It takes a 3D spatio-spectral object as input and produces a set of 2D projected data with multiple detectors of different characteristics. However, these 2D outputs suffer from non-stationary spatial and spectral blurring, as well as undersampling. Our first contribution is the development of the forward model in order to simulate data and the second is the use of this model to reconstruct a full 3D hyperspectral image from the projected measurements. This problem is ill-posed and we propose an algorithm based on the regularized leastsquare approach with convex edge-preserving regularization. We show on the simulation that our proposed model and algorithm allow a better reconstruction than the state of the art algorithm, thanks to spatial and spectral deconvolutions and denoising.
\end{abstract}

Index Terms - Hyperspectral - IFU - PSF - Superresolution - Dithering - Inverse Problem

\section{INTRODUCTION}

Hyperspectral imagery, which provides high-resolution spectra at many spatial positions, is widely used in remote sensing applications for multiple domains, such as astrophysics, medical diagnostics, military, food safety, etc [1,2]. To obtain a spatially resolved spectra projected on $2 \mathrm{D}$ detectors, Integral Field Units (IFU) instruments [3] are commonly adopted. They consist of observing the input through several thin slices (or microlens) in parallel, where each slice output is spectrally diffracted resulting in 2D measurements (one spatial dimension and one spectral dimension) [2]. These instruments, therefore, impose a stage of reconstruction.

\footnotetext{
* This project is funded by CNES. This work was performed using HPC resources from the "Mésocentre" computing center of CentraleSupélec and École Normale Supérieure Paris-Saclay supported by CNRS and Région Îlede-France (http://mesocentre.centralesupelec.fr/)
}

Several challenges arise in hyperspectral reconstruction, especially for long wavelengths $[4,5]$. First the optics, because of the diffraction, introduces a spatial blurring that depends on the wavelength. The object is more blurred at long wavelengths than at small wavelengths (see Sec. 2.1). Second, the wavelength dispersion introduces a spectral blurring that is also wavelength-dependent, resulting in non stationary blurring (see Sec. 2.3). Furthermore, in most cases, the signal is undersampled at the detector level, with different spatial and spectral sampling steps between detectors. To overcome undersampling, a so-called "dithering" method $[6,7]$ is adopted, consisting of using several pointings with small spatial shifts (see Sec. 2.4).

The standard algorithm for reconstruction of the $2 \mathrm{D}+\lambda$ object is based on co-addition methods, where the spatial and spectral blurring effects are neglected. However, in this work we are dealing with wide spectral bands in the infrared, which requires to take into account the blurring effects.

Our approach relies on the development of a new forward model of the data, that takes into account all the effects mentioned above. The reconstruction algorithm is based on regularized least square methods [8], where a data adequacy term models the instrument effect and a convex edge-preserving regularization term prevents noise amplification without excessive penalization of high spatial gradients [9]

\section{INSTRUMENT MODEL}

\subsection{Optic response}

The optical system is the first component of the model; it aims to focus sky images on the focal plane. The spatial resolution of the focused images is limited by diffraction with a spatial blur whose Point Spread Function (PSF) width varies linearly with wavelengths as illustrated in Fig. 1. In its Field of View (FOV) which has a limited size, the instrument can be considered spatially stationary. Thus, the result is a spatial convolution of the sky $\boldsymbol{x}[k, l, m]$ with $(k, l, m) \in[1, \ldots, K] \times$ $[1, \ldots, L] \times[1, \ldots M]$, by the PSF of the optics, denoted $\boldsymbol{h}$, 
that depends on the wavelength:

$$
\begin{aligned}
\boldsymbol{x}_{\mathrm{opt}}[k, l, m] & =\sum_{k^{\prime}, l^{\prime}} \boldsymbol{x}\left[k^{\prime}, l^{\prime}, m\right] \times \boldsymbol{h}\left[k-k^{\prime}, l-l^{\prime}, m\right] \\
& =\boldsymbol{x}_{k, l}^{*} \boldsymbol{h}
\end{aligned}
$$

For fast computations, the convolution is done using Discrete Fourier Transform, leading to circular spatial convolution [10]. This hypothesis is not an approximation since spatial edges are properly taken into account.

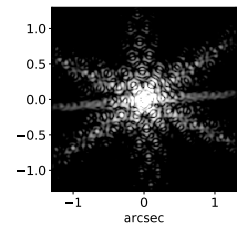

(a) $\lambda=5 \mu \mathrm{m}$

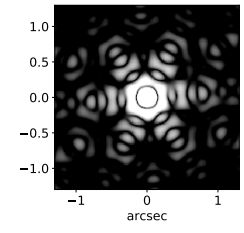

(b) $\lambda=15 \mu \mathrm{m}$

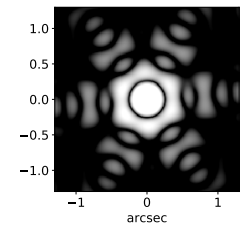

(c) $\lambda=25 \mu \mathrm{m}$
Fig. 1. PSF at different wavelengths of JWST/MIRI (logarithmic scale) simulated with the WebbPSF simulator [11].

\subsection{Spatial and spectral divisions}

A part of the blurred sky image is selected on the focal plane. This selection is spatially done by a set of slits and spectrally by wavelength filters [12]. Our model takes into account the spatial width and length of each slit and the spectral bandpass of each filter. In that way, the model writes

$$
\boldsymbol{x}_{i}[k, l, m]=\boldsymbol{x}_{\mathrm{opt}}[k, l, m] \times \boldsymbol{w}_{i}[k, l, m]
$$

where $i \in I$ means the spatial + spectral $i$-selection and $\boldsymbol{w}_{i}$ the corresponding window with

$$
\boldsymbol{w}_{i}[k, l, m]= \begin{cases}1, & \text { if }(k, l, m) \in \mathcal{S}_{i} \\ 0, & \text { otherwise, }\end{cases}
$$

$\mathcal{S}_{i}$ being the support of the selection $i$. The whole set of selections $I$ takes into account all the slits, combined with the different spectral channels and the successive pointings. The light is then directed towards the diffraction gratings.

\subsection{Diffraction gratings}

Ideally, the gratings output for a monochromatic point source is a Dirac on the detector whose position depends only on the wavelength. However, gratings are not perfect and the response (or the spectral PSF) for a monochromatic point source [13] at wavelength $\lambda$ writes

$$
h_{r}(u ; s, \lambda)=B \operatorname{sinc}^{2}\left(\pi W\left(\frac{u-c \times s}{\lambda}-\frac{1}{a}\right)\right)
$$

(a)

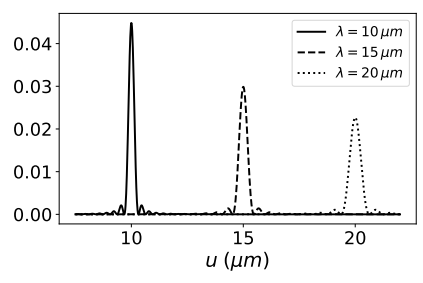

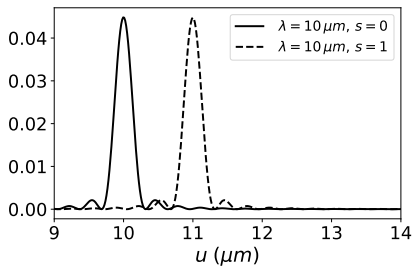

(b)
Fig. 2. (a) Spectral dependence of the grating response,(b) influence of the spatial position of the input source, $s$, on the position of the diffracted light.

where $u$ is a spatial position on the detector (in wavelength unit), $s$ the spatial position of the input source, and $c$ the calibration factor which converts the spatial position into wavelength. The spectral PSF is non-stationary, due to the width of the cardinal sin which increases linearly with the wavelength as shown in Fig. 2(a). Moreover, it also depends on the spatial position $s$ in the slice, which influences the position of the dispersed light projected onto the detector as shown in Fig. 2(b). This degeneracy is one reason to split the light with slits.

The spectral PSF (Eq. 4) is parametrized by $W$ that is related to the length of the grating, and the grating step $a$. The former controls the spectral width, hence the spectral resolution. We suppose that the instrument is properly calibrated, so $W$ can be derived from the spectral resolution instrument specification $R=\lambda / \Delta \lambda$, where $\Delta \lambda$ is the FWHM of the spectral PSF at wavelength $\lambda$. Basic calculus leads to $W \approx 2.8 R / \pi$. The other parameter $a$ controls the position of the spectral PSF on the detector. In practice $a=1$ for calibrated measurements.

Finally, the gratings are supposed linear and non-invariant, leading to the output of the gratings $\boldsymbol{g}_{i}$, for each selection $i$, expressed as

$$
\boldsymbol{g}_{i}\left[k, m^{\prime}\right]=\sum_{l \in \mathcal{L}_{i}} \sum_{m \in \mathcal{M}_{i}} \boldsymbol{x}_{i}[k, l, m] \times \boldsymbol{h}_{r, i}\left[m^{\prime} ; l, m\right]
$$

where $\boldsymbol{h}_{r, i}\left[m^{\prime} ; l, m\right]=h_{r}\left(u_{m^{\prime}} ; s_{l, i}, \lambda_{m, i}\right)$ is the spectral PSF (Eq. (4)) for the spatial position $s_{l}$ inside slit $i$, and the wavelength $\lambda_{m, i}$ inside slit $i$.

The 2D output $\boldsymbol{g}_{i}$ depends on a spatial dimension $k$ and a spectral dimension $m^{\prime} \in\left[1, \ldots, M^{\prime}\right]$, with $M^{\prime}$ the number sampled wavelengths on the detector.

\subsection{Spatial sampling and "dithering"}

The sampling step of the input $\boldsymbol{x}$ and of the detector are different. In particular, the spatial sampling step on the detector and the slit width are often too large, resulting in under-sampling and aliasing. To compensate, "dithering" is used. It consists of using several pointings with small spatial shifts, leading to a super-resolution problem for data processing. Furthermore, 
the spatial sampling depends on the detector and the selection $i$ (corresponding to a specific wavelength range, slit and pointing direction).

In the previous section, the spectral sampling step along the $m$ axis, and the spatial sampling step along the $l$ axis (slit width) are modeled in Eq. (5). For the other spatial dimension, $k$, we suppose that the detector sampling step is a multiple $N_{i}$ of the input sampling step. The data then writes

$$
\boldsymbol{y}_{i}\left[k^{\prime}, m^{\prime}\right]=\sum_{k=k^{\prime} N_{i}}^{\left(k^{\prime}+1\right) N_{i}} \boldsymbol{g}_{i}\left[k, m^{\prime}\right] .
$$

Concretely, this summation is computed by convolution with a square impulse response of size $N_{i}$, followed by subsampling every $N_{i}$. Note that the spatial resolution of the input is arbitrary chosen to minimize the error on pixel size of the detector and the exact pointing position.

\subsection{Complete model}

The instrument model establishes a relationship between $\boldsymbol{x}[k, l, m]$ and $\boldsymbol{y}_{i}\left[k^{\prime}, m^{\prime}\right]$ for a given selection $i$

$$
\begin{array}{r}
\boldsymbol{y}_{i}\left[k^{\prime}, m^{\prime}\right]=\sum_{k=k^{\prime} N_{i}}^{\left(k^{\prime}+1\right) N_{i}}\left(\sum_{l \in \mathcal{L}_{i}} \sum_{m \in \mathcal{M}_{i}}[\boldsymbol{x}[k, l, m] * \boldsymbol{h}[k, l, m]]\right. \\
\left.\boldsymbol{w}_{i}[k, l, m] \times \boldsymbol{h}_{r, i}\left[m^{\prime} ; l, m\right]\right)
\end{array}
$$

The model is linear but non-stationary, and can be summarized as $\boldsymbol{y}_{i}=\boldsymbol{H}_{i} \boldsymbol{x}$. Then, all data are concatenated giving $\boldsymbol{y}=\boldsymbol{H} \boldsymbol{x}$ with $\boldsymbol{y}^{t}=\left[\boldsymbol{y}_{1}^{t}, \boldsymbol{y}_{2}^{t}, \ldots, \boldsymbol{y}_{I}^{t}\right]$ and $\boldsymbol{H}^{t}=$ $\left[\boldsymbol{H}_{1}^{t}, \boldsymbol{H}_{2}^{t}, \ldots, \boldsymbol{H}_{I}^{t}\right]$ the full data model. This model takes into account several instrument effects:

- the spatial blurring depending on the wavelength,

- the different spectral channels with different number of slits of different sizes,

- the spectral blurring with varying grating parameters,

- the spatial and spectral sampling, with steps specific for each detector and different than the sampling steps of the input sky.

\section{RECONSTRUCTION}

\subsection{Standard method}

The state of the art algorithm for hyperspectral image reconstruction is based on co-addition (after preprocessing step of raw measures), which is the shift-and-add Super-Resolution algorithm (SR) [7, 14]. This method corresponds to the minimization of the least-square criterion

$$
\begin{aligned}
\hat{\boldsymbol{x}}_{\text {coadd }} & =\underset{\boldsymbol{x}}{\arg \min }\|\boldsymbol{y}-\boldsymbol{S} \boldsymbol{x}\|^{2} \\
& =\left(\boldsymbol{S}^{t} \boldsymbol{S}\right)^{-1} \sum_{i} \boldsymbol{S}_{i}^{t} \boldsymbol{y}_{i}
\end{aligned}
$$

where $\boldsymbol{S}$, a sub-sampling matrix, is equivalent to our model of Eq. (7), but with Dirac as spatial and spectral PSF, and without any spectral upsampling. In that case, $\boldsymbol{S}_{i}^{t}$ is the upsampling spatial matrix for a given pointing and slit and $\left(\boldsymbol{S}^{t} \boldsymbol{S}\right)^{-1}$ is a diagonal normalization matrix that counts the number of time a pixel is measured. This algorithm works with data that have identical spectral sampling step.

\subsection{Proposed method}

Our approach relies on (1) the complete forward model Eq. (7) for different channels, sampling steps, pointings, spatial and spectral blurring, and (2) the optimization of a mixed criterion that combines a data fidelity term and a regularization term. The reconstruction approach with the least-square method described in Sec. 3.1 suffers from the ill-conditioning coming from the blurring effects. Therefore, we enforce a regularization term based on edge-preserving convex potential [15]. Thus, the reconstructed cube $\hat{\boldsymbol{x}}$ is expressed as

$$
\begin{aligned}
\hat{\boldsymbol{x}}= & \underset{\boldsymbol{x}}{\arg \min }\|\boldsymbol{y}-\boldsymbol{H} \boldsymbol{x}\|^{2}+ \\
& \mu_{\text {spec }} \sum_{w \in \mathcal{W}} \phi\left(\boldsymbol{d}_{w}^{t} \boldsymbol{x} ; T_{\text {spec }}\right)+\mu_{\text {spat }} \sum_{i \in \mathcal{I}} \phi\left(\boldsymbol{d}_{i}^{t} \boldsymbol{x} ; T_{\text {spat }}\right)
\end{aligned}
$$

where $\boldsymbol{d}_{w}$ computes the first order differences along the spectral dimension, $\boldsymbol{d}_{i}$ the first order differences in row and column along the spatial dimension, $\mu_{\text {spec }}$ and $\mu_{\text {spat }}$ the spectral and spatial regularization parameters respectively, and $\phi(\delta, T)$ the Huber potential defined as

$$
\phi(\delta, T)= \begin{cases}\delta^{2}, & \text { if }|\delta| \leq T \\ 2 T|\delta|-T^{2} & \text { otherwise }\end{cases}
$$

Huber potential is continuously differentiable, with a quadratic behavior below the threshold $T$ to enforce denoising, and a linear behavior above the threshold to prevent excessive penalization at high gradient values and to preserve edges. The closed form expression of the minimizer $\hat{\boldsymbol{x}}$ is not explicit and cannot be directly calculated. Therefore, we rely on the fast semi-quadratic (or majorize-minimize) algorithm based on the Geman and Yang structure to find the solution [9].

\section{SETUP AND RESULTS}

\subsection{Setup for the experiment}

Our instrument model is based on the Mid Resolution Spectrometer (MRS) of the Mid-Infrared Instrument (MIRI) [12] 
on board the JWST. We reproduce hyperspectral data with our instrument model for a given "Urban" [16] object represented on a 3D Cartesian grid with $K \times L \times M=120 \times 120 \times 2000$ pixels. This object is assumed to be spatially sampled with a step size $\Delta_{k}=\Delta_{l}=0.1 \mathrm{arcsec} / \mathrm{pixel}$. The spectral dimension is uniformly sampled from $7.4 \mu \mathrm{m}$ to $18 \mu \mathrm{m}$, and is divided into two spectral channels observed simultaneously. Furthermore, the IFU divides the $l$-axis into slices with a width equal to $3 \times \Delta_{l}$ for the first channel and $4 \times \Delta_{l}$ for second channel. The light in each slice is projected onto different detectors with a pixel step size equal to $2 \times \Delta_{k}$ and $3 \times \Delta_{k}$ for to the first and second channels, respectively. We take a dithering pattern of 8 pointing directions. For a single pointing, each diffracted slice is a $2 \mathrm{D}$ spatio-spectral image of size $21 \times 250$ pixels and $19 \times 250$ for the first channel and the second channel, respectively. The set of $2 \mathrm{D}$ output is then corrupted with an additive zero-mean white Gaussian noise, and with $\mathrm{SNR}=30 \mathrm{~dB}$.

The proposed reconstruction is computed using Eq. 8 . Hyperparameters are set to $T_{\text {spat }}=0.5, T_{\text {spec }}=2.5, \mu_{\text {spat }}=$ $0.5, \mu_{\mathrm{spec}}=1.5$ by minimizing the reconstruction error $E(\hat{\boldsymbol{x}})=\left\|\boldsymbol{x}^{*}-\hat{\boldsymbol{x}}\right\|^{2}$ where $\boldsymbol{x}^{*}$ is the true sky. The algorithm is implemented in python with the numpy toolbox.

\subsection{Results and discussion}

Fig. 3 shows a spatial reconstruction for $\lambda=9 \mu \mathrm{m}$ and $\lambda=$ $15 \mu \mathrm{m}$. These wavelengths belong to two different spectral channels. We have reconstructed a unique cube, with a FOV of $6.4 \times 7.2$ arcsec for the first channel and $7.8 \times 9.3$ arcsec for the second one. The standard and proposed algorithms show a better reconstruction at small wavelength (first column) than at long wavelength (second column) since the object is spatially less blurred.

The standard method reconstructs large scale patterns, but fails to reproduce small scale structures because the blurring is not taken into account; the relative error is $E_{\text {standard }}=7 \%$. With the proposed approach, the spatial resolution is significantly improved, small scale details are detected, and the error is reduced to $E_{\text {proposed }}=3.5 \%$. Fig. 4 compares the spectra of two single pixels for the input, the standard reconstruction, and the proposed reconstruction. We see that the proposed algorithm minimizes the spectral mixing via deconvolution and allows denoising.

\section{CONCLUSION}

We have developed a forward model of IFU instruments, establishing a relationship between a $3 \mathrm{D}$ spatio-spectral input and a set of 2D spatio-spectral outputs projected onto different detectors. This model takes into account (1) the spatial and spectral blurring that depends on the wavelength, (2) the different spectral channels with a different number of slits of different sizes, and (3) the irregular under-sampling. The loss
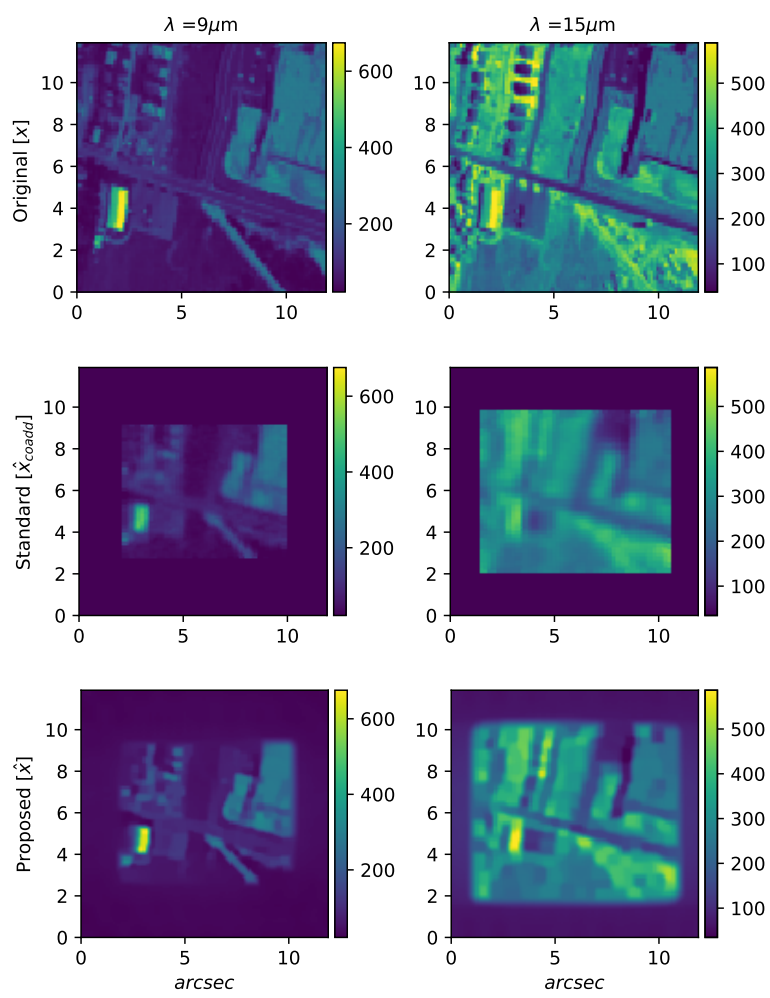

Fig. 3. Top: observed input image at $\lambda=9 \mu \mathrm{m}$ and $\lambda=$ $15 \mu \mathrm{m}$. Middle: standard reconstruction at the same wavelengths, Bottom: proposed reconstruction $\hat{\boldsymbol{x}}$.

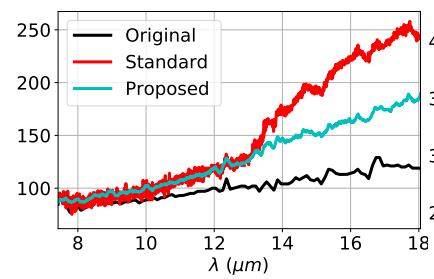

(a) Pixel $(70,75)$

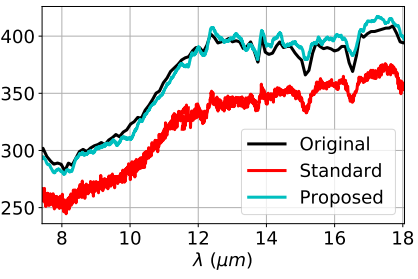

(b) Pixel $(50,75)$
Fig. 4. Comparison between the spectra of two chosen pixels for the observed input, the standard and the proposed reconstruction.

of spatial information is compensated by several acquisitions with small spatial shifts ("dithering" strategy). Our reconstruction algorithm is based on the least square methods with edge-preserving convex Huber regularization. It reconstructs more spatial details at a small scale compared to the standard algorithm, as well as spectral deconvolution and denoising.

Without any optimization, the proposed algorithm actually takes one hour to reconstruct the $120 \times 120 \times 2000$ hyperspectral image with a single CPU at $5 \mathrm{GHz}$ with $32 \mathrm{~GB}$ of memory, whereas the standard algorithm takes a few seconds. 


\section{REFERENCES}

[1] M. J. Khan, H. S. Khan, A. Yousaf, K. Khurshid, and A. Abbas, "Modern trends in hyperspectral image analysis: A review," IEEE Access, vol. 6, pp. 14118-14129, 2018.

[2] Fabrizio Vagni, "Survey of hyperspectral and multispectral imaging technologies (etude sur les technologies d'imagerie hyperspectrale et multispectrale," p. 44, 052007.

[3] S. Vives, E. Prieto, Y. Salaun, and P. Godefroy, "New technological developments in integral field spectroscopy," in Advanced Optical and Mechanical Technologies in Telescopes and Instrumentation, Eli AtadEttedgui and Dietrich Lemke, Eds. International Society for Optics and Photonics, 2008, vol. 7018, pp. $959-$ 968, SPIE.

[4] M. A. Hadj-Youcef, F. Orieux, A. Fraysse, and A. Abergel, "Restoration from multispectral blurred data with non-stationary instrument response," in 2017 25th European Signal Processing Conference (EUSIPCO), Aug 2017, pp. 503-507.

[5] T Rodet, François Orieux, Jean-François Giovannelli, and Alain Abergel, "Data inversion for hyperspectral objects in astronomy," 092009 , pp. $1-4$.

[6] A. S. Fruchter and R. N. Hook, "Drizzle: A method for the linear reconstruction of undersampled images," Publications of the Astronomical Society of the Pacific, vol. 114, no. 792, pp. 144-152, feb 2002.

[7] R. Hook and Andrew Fruchter, "Dithering, sampling and image reconstruction,” vol. 216, pp. 521, 012000.

[8] G. Demoment, "Image reconstruction and restoration: overview of common estimation structures and problems," IEEE Transactions on Acoustics, Speech, and Signal Processing, vol. 37, no. 12, pp. 2024-2036, Dec 1989.

[9] J. Idier, "Convex half-quadratic criteria and interacting auxiliary variables for image restoration," IEEE Transactions on Image Processing, vol. 10, no. 7, pp. 10011009, July 2001.

[10] B. Bamieh, "Discovering the fourier transform: A tutorial on circulant matrices, circular convolution, and the dft," 052018.

[11] Marshall D. Perrin, Rémi Soummer, Erin M. Elliott, Matthew D. Lallo, and Anand Sivaramakrishnan, "Simulating point spread functions for the James Webb Space Telescope with WebbPSF," in Space Telescopes and Instrumentation 2012: Optical, Infrared, and Millimeter
Wave, Mark C. Clampin, Giovanni G. Fazio, Howard A. MacEwen, and Jacobus M. Oschmann Jr., Eds. International Society for Optics and Photonics, 2012, vol. 8442, pp. 1193 - 1203, SPIE.

[12] Martyn Wells, J W. Pel, A Glasse, Gillian Wright, Gabby Kroes, Ruyman Azzollini, Steven Beard, Bernhard Brandl, Angus Gallie, V C. Geers, A M. Glauser, Peter Hastings, Th Henning, Rieks Jager, Kay Justtanont, Bob Kruizinga, Fred Lahuis, David Lee, Ismael Martinez Delgado, and David Wright, "The midinfrared instrument for the james webb space telescope, vi: The medium resolution spectrometer," Publications of the Astronomical Society of the Pacific, vol. 127, pp. 646-664, 072015.

[13] Joseph W Goodman, Introduction to Fourier Optics McGraw-Hill Series in Electrical and Computer Engineering, vol. 8, 1996.

[14] Sung Cheol Park, Min Kyu Park, and Moon Gi Kang, "Super-resolution image reconstruction: a technical overview," IEEE Signal Processing Magazine, vol. 20, no. 3, pp. 21-36, May 2003.

[15] M. Nikolova and M. Ng, "Fast image reconstruction algorithms combining half-quadratic regularization and preconditioning," in Proceedings 2001 International Conference on Image Processing (Cat. No.01CH37205), vol. 1, pp. 277-280 vol.1, ISSN: null.

[16] Feiyun Zhu, Ying Wang, Shiming Xiang, Bin Fan, and Chunhong Pan, "Structured sparse method for hyperspectral unmixing," ISPRS Journal of Photogrammetry and Remote Sensing, vol. 88, pp. 101-118, 2014. 